\title{
Joint Design of Vector Quantizers and RCPC Channel Codes for Rayleigh Fading Channels
}

\author{
Yirong Shen ${ }^{\dagger *}$ Andrea J. Goldsmith ${ }^{\dagger}$, and Michelle Effros ${ }^{\ddagger}$ \\ †Stanford University \\ ${ }^{\ddagger}$ California Institute of Technology
}

\begin{abstract}
We study the performance of joint source and channel codes designed to minimize end-to-end distortion over a Rayleigh fading channel. We consider two joint code designs. The first joint code uses a sequential design: a standard vector quantizer (VQ) source code is designed for a perfect channel (noiseless and distortionless) and then an RCPC channel code is optimized relative to the VQ and the channel statistics. The second design jointly optimizes a channel optimized VQ (COVQ) and an RCPC channel code through an iterative design process. We consider both hard-decision and soft-decision decoding for the channel codes. In both designs the bit allocation between the source and channel codes is optimized. At this optimal bit allocation, the performance of the iterative joint design and the simpler sequential design are nearly the same over the range of SNR values that we considered. Both code designs outperform standard COVQ by up to $6 \mathrm{~dB}$, and this performance improvement is most pronounced at low SNRs.
\end{abstract}

\section{Introduction}

In this work we extend the joint source and channel code designs applied to AWGN channels in [1] to Rayleigh fading channels. These joint designs use vector quantization (VQ) for the source code and RCPC coding for the channel code with either hard decision (HD) or soft decision (SD) decoding. In general, HD decoding performs worse than SD in exchange for reduced complexity.

We consider both sequential and iterative joint source and channel code designs. The sequential design has less complexity than the iterative design since the source code is designed independently of the channel. Specifically, in our sequential design the VQ is based on a perfect channel model, i.e. a channel that does not introduce any noise or distortion. The RCPC code is then optimized with respect to this source code and

\footnotetext{
*The work of Y. Shen was partially supported by the Stanford summer undergraduate research program.
}

the channel signal-to-noise power (SNR) to minimize end-to-end distortion. The VQ used in the iterative design is a Channel-Optimized Vector Quantizer (COVQ) that has been optimized for the index crossover probabilities of the RCPC channel code design. The designs of the COVQ and RCPC codes are not independent. The optimal COVQ is the COVQ matched to the index crossover probabilities determined by the RCPC channel code and the channel. Likewise, the optimal RCPC code is the RCPC code that minimizes the expected distortion of the COVQ. Ideally, the COVQ and the RCPC code should be designed simultaneously. However, this is difficult to do in practice. Therefore, we optimize the RCPC and COVQ successively using an iterative descent technique. Both of our design algorithms optimize the bit allocation between the source and channel codes for the given SNR.

For both designs we obtain their average end-toend distortion over a range of channel SNR values ${ }^{1}$. We compare this distortion with that of standard COVQ without additional channel coding. We further examine this distortion to determine how much is contributed by the source code versus the channel distortion. Surprisingly, even at very low channel SNRs, at the optimal bit allocation the distortion is mainly contributed by the source code. The same was true of the optimal joint source and channel code design for AWGN channels [1].

The remainder of this paper is organized as follows. The end-to-end system model is presented in Section 2. The COVQ, RCPC channel codes, and joint iterative code design are described in Section 3. Experimental results appear in Section 4. Our conclusions are given in Section 5 .

\section{System Model}

A block diagram of the end-to-end communication system is shown in Figure 1. This model differs from that in [1] by the addition of amplitude fading $a(t)$. We assume a discrete-time, real-valued, stationary source. The

\footnotetext{
${ }^{1}$ Results on the distortion of the iterative code design for a 10 $\mathrm{dB}$ channel SNR were reported in [2].
} 
source encoder $\alpha$ maps the set of possible $k$-dimensional source vectors $\underline{x}$ into a set of binary strings $\underline{v}$ of fixed length $k R_{s}$. The number of bits per source symbol, $R_{s}$, is a parameter of the source code design. The channel encoder $\phi$ operates on the vector $\underline{v}$ of $k R_{s}$ bits to obtain $k\left(R_{s}+R_{c}\right)$ coded bits $\underline{w}$. These coded bits (channel symbols) are interleaved, modulated using binary phase shift keying (BPSK) and then transmitted over the channel at a rate of one channel symbol per $T$ seconds, where $T$ is the channel symbol time.

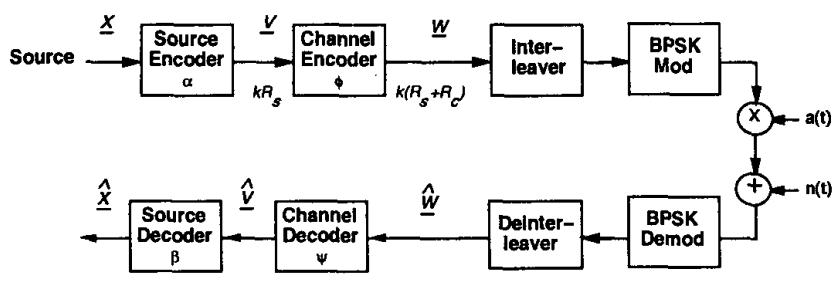

Figure 1: System Model.

The channel has time-varying amplitude fading $a(t)$, AWGN $n(t)$, and no intersymbol interference. In our numerical results we assume the amplitude variations follow a Rayleigh distribution: $p(a)=$ $2 a \exp \left[-a^{2}\right], a>0$, but our joint code design can be applied to any fading distribution for which the bit-errorprobability is known. The interleaver/deinterleaver pair are assumed to have infinite depth so that the amplitude values appear i.i.d. to the channel decoder.

At the receiver, the signal is first demodulated and then deinterleaved, which yields the noisy channel symbols $\underline{\hat{w}}$. These channel symbols are passed through the channel decoder $\psi$, which may correct some (or all) of the channel errors to obtain a noisy reproduction $\underline{\hat{v}}$ of the original binary string $\underline{v}$. Finally, $\underline{\hat{v}}$ is passed through the source decoder $\beta$ to obtain a noisy reproduction $\underline{\hat{x}}$ of the original source vector $\underline{x}$.

The total end-to-end distortion of the system is the mean-squared-error between the source vector $\underline{x}$ and source reproduction vector $\underline{\hat{x}}: d(\underline{\underline{x}}, \underline{\hat{x}})=\|\underline{x}-\underline{\hat{x}}\|^{2}$. For a given $R_{s}$, the total distortion decreases as $R_{c}$ increases, since the channel code has more redundancy and can better protect against channel errors. Similarly, for a given $R_{c}$, the total distortion decreases as $R_{s}$ increases, since the source code has more bits to represent the original source vector. For $R=R_{s}+R_{c}$ fixed, there is an optimal way to divide the transmission rate $R$ between the source and channel rates $R_{s}$ and $R_{c}$ to minimize total distortion. This optimal allocation depends on the SNR per channel symbol $E_{s} / N_{0}$, the fading distribution, the channel code, and the source statistics. For example, channel coding is less important on high-SNR channels, so the $R_{s}$ value corresponding to the optimal bit allocation will generally increase as a function of $E_{s} / N_{0}$. For our joint code designs we consider all
$\left(R_{s}, R_{c}\right)$ pairs such that $R_{s}+R_{c}=R$ and choose the pair and corresponding joint code with minimal distortion. In our joint code designs for AWGN channels the optimal bit allocation turned out to be the most important aspect of the code design [1], and we will see that the same is true for fading channels. In particular, the iterative and sequential code designs have roughly the same performance as long as the bit allocation is optimized.

\section{Joint Code Design Algorithms}

In this section we briefly describe our sequential and iterative joint source and channel code design algorithms: more details can be found in $[1,2]$. The iterative design will always have an expected distortion at least as low as the expected distortion of the sequential design, since the codes resulting from the sequential design are considered during the joint iterative design process and improved through this process when possible. We therefore begin by describing the joint iterative design algorithm. We will then describe the sequential technique, which is a subset of this algorithm.

\subsection{Joint Iterative Design (COVQ- RCPC)}

The goal of our joint code design is to minimize the expected distortion $D=\mathrm{E}[d(\underline{x}, \underline{\hat{x}})]$ of the COVQ and RCPC codes, where the expectation is with respect to the source data training set and the channel statistics. We use an iterative design technique to obtain this minimization. Specifically, for a given $R_{s}$ value we alternately optimize the COVQ for a given RCPC code and then the RCPC code for the new COVQ.

The flow chart for our design algorithm for a fixed $R_{s}$ value is shown in Figure 2. The design algorithm consists of three steps. In Step 0 we initialize the system such that $\operatorname{Pr}(\underline{\hat{v}}=\underline{v} \mid \underline{v})=1$. This initialization is equivalent to assuming a perfect channel. In Step 1 of the design algorithm, the COVQ $(\alpha, \beta)$ is optimized for the bit-error-probability of the given RCPC channel code to minimize the expected distortion $D=E[d(\underline{X}, \underline{\hat{X}})]$ between a random source input vector $\underline{X}$ and its reproduction $\underline{\hat{X}}$ at the receiver. The bit-error-probabilities result either from the initialization of Step 0 or from the channel $E_{s} / N_{0}$, the channel fading statistics, and the RCPC channel code $(\phi, \psi)$ found in Step 2. The optimal $\alpha$ and $\beta$ are obtained through the COVQ design algorithm described in [3].

In Step 2 of the algorithm, the RCPC code with minimal distortion for the COVQ designed in Step 1 is found. We use the set of channel code rates from Table 1 in [4] to obtain our RCPC code candidates. Each candidate RCPC code provides a set of $k R_{s}$ biterror-probabilities for the $k R_{s}$ bits in $\underline{v}$. We repre- 
sent each candidate code by a vector of length $k R_{s}$, where the $j$ th vector element corresponds to the channel code rate applied to the $j$ th bit in $\underline{v}$. Associated with the convolutional code applied to the $j$ th bit is a bit-error-probability $P_{j}$ which depends on the channel $E_{s} / N_{0}$, the channel fading statistics, and the decoder assumptions (hard or soft decision, channel side information, etc). These bit-error-probabilities were derived in [4] for Rayleigh fading channels assuimg HD decoding and no channel side information [4, Eqn. 11] and SD decoding with channel side information [4, Eqn. 13]. We use these bit-error-probabilities to determine the performance of our joint source and channel code designs under either SD or HD channel decoding. The minimal-distortion RCPC code is obtained, for a given COVQ and corresponding $R_{s}$ value, by searching over all $\mathrm{RCPC}$ code vectors that satisfy the transmission rate constraint $R=R_{s}+R_{c}$. The optimal RCPC code is the code with the error protection levels that minimize the expected distortion while satisfying this transmission rate constraint.

At the conclusion of Step 2 a new channel code $(\phi, \psi)$ has been designed for the COVQ obtained in Step 1. The iterative design process then returns to Step 1 to determine the COVQ $(\alpha, \beta)$ for this new RCPC channel code $(\phi, \psi)$. Once a new COVQ is obtained, this source code is passed to Step 2 of the algorithm to obtain a new RCPC channel code. Successive application of Steps 1 and 2 results in a sequence of source codes $\{(\alpha, \beta)\}$ and corresponding channel codes $\{(\phi, \psi)\}$ for which the expected distortions form a positive nonincreasing sequence which has to converge to a locally optimal solution, since each design step gives a global optimum. At convergence, the source code $(\alpha, \beta)_{R_{s}}^{*}$ and channel code $(\phi, \psi)_{R_{s}}^{*}$ with minimal distortion for the given $R_{s}$ value are obtained. The design process is repeated for each $R_{s}$ value $0 \leq R_{s} \leq R$, and the source code $(\alpha, \beta)^{*}$ and channel code $(\phi, \psi)^{*}$ corresponding to the $R_{s}$ value with minimal distortion comprise the final joint code. For $R_{s}=0$, the source is represented by a single codeword, so there is no channel distortion and the total distortion corresponds to the source variance. For $R_{s}=R$, all redundant bits are allocated to the source code, so there is no channel coding, i.e. $R_{c}=0$. This corresponds to standard COVQ [3].

\subsection{Sequential Code Design (VQ- RCPC)}

Our second joint design is a sequential design which uses a VQ designed for a perfect channel followed by an RCPC channel code matched to the VQ to minimize distortion. For a given value of $R_{s}$ this source and channel code is designed by following Steps 0-2 of Figure 2 and then stopping, so the iterative design process is eliminated. This process is repeated for each $R_{s}$

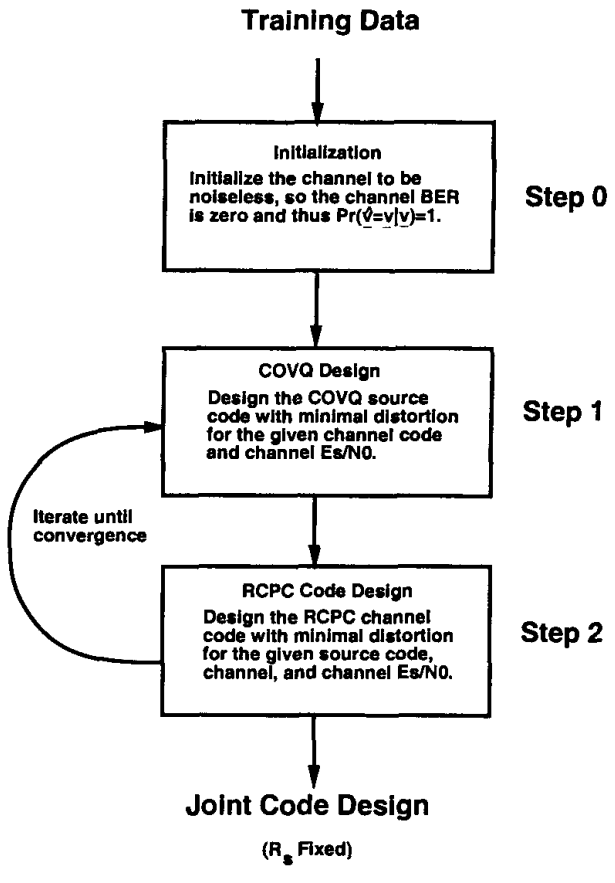

Figure 2: Iterative Code Design Process.

value, $0 \leq R_{s} \leq R$, and the joint source and channel code corresponding to the $R_{s}$ value with minimal distortion is used for the final joint code. The advantage of this technique relative to the joint iterative design is its simplicity: the source code design is independent of all aspects of the channel code, modulation, and channel SNR.

\section{Experimental Results}

The joint source and channel code designs described in Section 3 were implemented and run for a range of channel $E_{s} / N_{0}$ values. Our experimental results are computed for a test data set of 5 magnetic resonance images (MRIs) applied to the joint code designed from a training data set of 20 other MRIs. The same data set was used to obtain the numerical results in [1]. We define the ratio of signal power to quantization and channel noise power (SQCNR) as $\operatorname{SQCNR}(\mathrm{dB})=10 \log \left(\sigma^{2} / D\right)$, where $D$ is the distortion of the joint code averaged over the test data set and the channel statistics, and $\sigma^{2}$ is the distortion of a rate zero $\left(R_{s}=0\right)$ VQ averaged over the test data set. We use vector dimension $k=4$ and consider $0 \leq R_{s} \leq 2$ bits per pixel (bpp) and $E_{s} / N_{0}$ of $5,7.5,10,12.5$, and $15 \mathrm{~dB}$.

Figure 3 shows the performance of the two joint design algorithms for both $\mathrm{HD}$ and $\mathrm{SD}$ decoding of the RCPC channel codes as well as the performance of the standard COVQ with no additional channel coding 


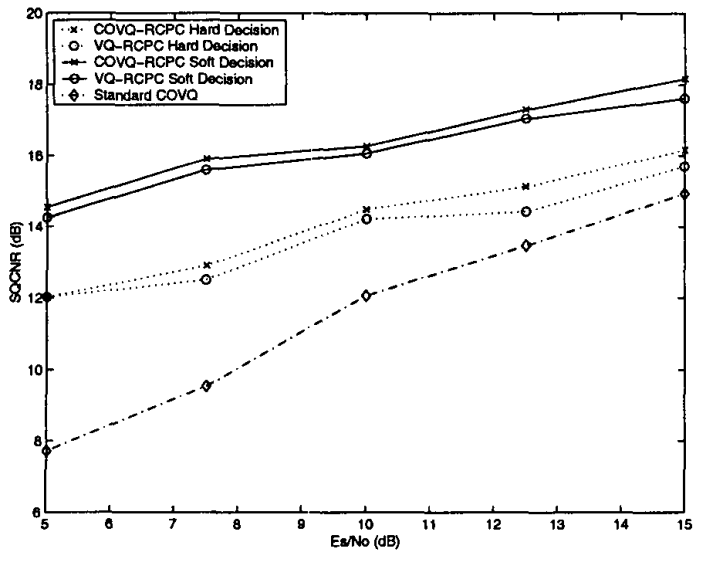

Figure 3: SQCNR versus $E_{s} / N_{0}$ at optimal $R_{s}$.

$\left(R_{c}=0\right)$. As expected, the SQCNR increases monotonically as $E_{s} / N_{0}$ increases. Notice that the joint design algorithms exhibit up to $4 \mathrm{~dB}$ of performance gain over COVQ for HD channel decoding and up to $6 \mathrm{~dB}$ of gain for SD channel decoding. Notice also that the performance of the iterative and sequential design techniques is nearly the same, with the joint iterative design (COVQ-RCPC) performing slightly better than the sequential design (VQ-RCPC) in all cases. The superiority of the COVQ-RCPC over VQ-RCPC is expected, but the minimal performance difference between the two is somewhat surprising. To understand the similarity in performance of our joint code designs, we now investigate the performance of the two algorithms in more detail.
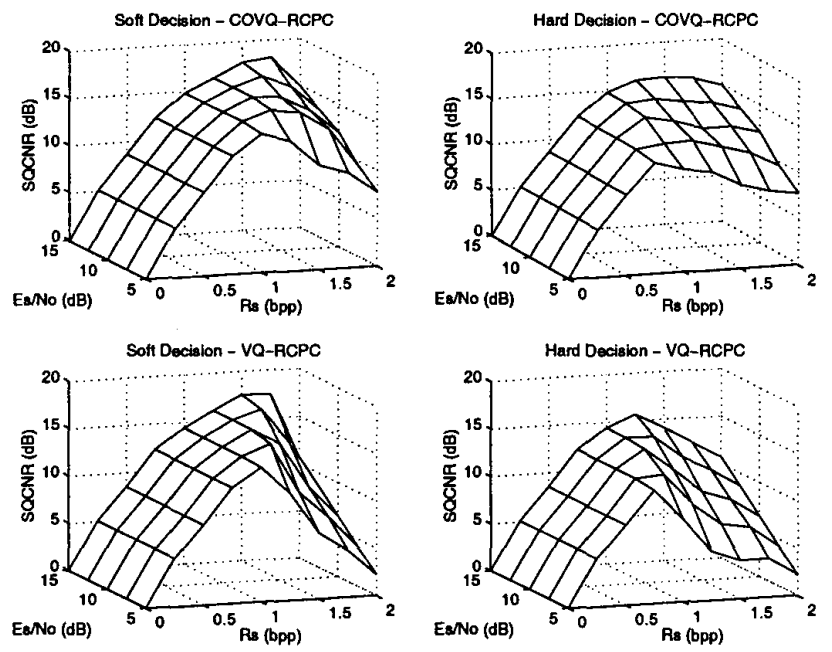

Figure 4: SQCNR versus $E_{s} / N_{0}$ and $R_{s}$.

In Figure 4 we show three-dimensional plots of SQCNR as a function of the bit allocation $R_{s}$ and the channel $E_{s} / N_{0}$ for the two design algorithms. Some of the $R_{s}$ values (e.g. $R_{s}=0$ ) would never be used in a real code design. However, a reasonable but improper choice of the bit allocation can significantly reduce the SQCNR. For example, choosing $R_{s}=.5$ or 2 bpp results in a $9 \mathrm{~dB}$ reduction in SQCNR for SD decoding and a $7 \mathrm{~dB}$ reduction for $\mathrm{HD}$ decoding. As expected, the $R_{s}$ value that maximizes SQCNR (minimizes distortion) increases as the channel $E_{s} / N_{0}$ increases, since fewer redundant bits are needed for channel coding. This trend is illustrated more clearly in Figure 5, where we plot the optimal $R_{s}$ as a function of $E_{s} / N_{0}$. An exception to this trend occurs at $E_{s} / N_{0}=7.5 \mathrm{~dB}$ for COVQ-RCPC using HD decoding. This exception is due to the fact that allocating the channel code additional redundancy at this $E_{s} / N_{0}$ sharply reduces its bit-error-probability, which is enough to compensate for the additional distortion in the source code entailed by reducing $R_{s}$.

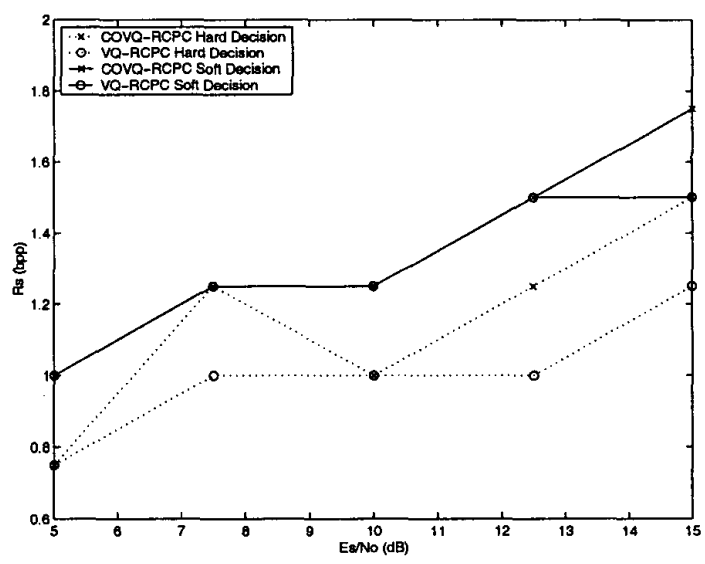

Figure 5: Optimal $R_{s}$ versus $E_{s} / N_{0}$.

A comparison of SQCNR versus $R_{s}$ is shown in Figure 6 for $E_{s} / N_{0}=5,7.5,10,12.5$, and $15 \mathrm{~dB}$. We see that the optimal bit allocation for both design algorithms is almost the same for HD decoding and for $\mathrm{SD}$ decoding. Note that the optimal $R_{s}$ value for the COVQ-RCPC code is always greater than or equal to that of the VQ-RCPC code, and the optimal $R_{s}$ value for SD decoding is always greater than or equal to that of HD decoding. This result is expected since for VQRCPC the source encoder is designed with respect to a perfect channel. Thus the encoded bits are more sensitive to channel errors, and therefore need stronger channel coding for a higher level of error protection. Also, SD channel decoding has better error-correcting performance than HD decoding, and therefore the joint codes using SD decoding will require less redundancy allocated to the channel code. Similarly, more powerful channel codes (e.g. turbo codes) would likely have smaller optimal $R_{s}$ values than the RCPC codes. As is 

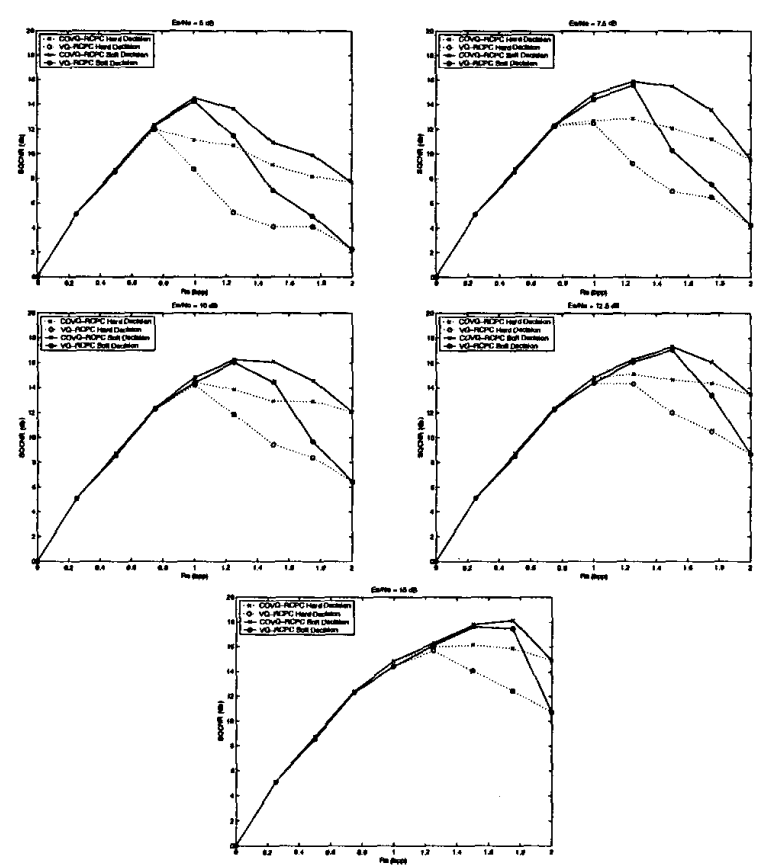

Figure 6: SQCNR versus $R_{s}$ for all Design Techniques.

evident from Figure 6, the most important aspect of a joint source and channel code design is choosing the appropriate bit allocation between the source and channel codes. Note that the SQCNR for the VQ-RCPC code designs falls off sharply as $R_{s}$ increases above its optimal value, whereas COVQ-RCPC is less sensitive to a sub-optimal $R_{s}$ choice. This same behavior was exhibited in the joint code performance on AWGN channels.

The distortion $\mathrm{D}$ at $E_{\mathrm{s}} / N_{0}=10 \mathrm{~dB}$ for each technique is shown by the solid lines in Figure 7 . The dotted lines in Figure 7 show the distortion contribution of the source code alone, based on the test data set and a perfect channel (we assume that the source encoder design is based on a fading channel with the given SNR). Note that for both design techniques and both SD and HD decoding, most of the distortion at the optimal bit allocation is contributed by the source code. Similar distortion results were obtained for the other $E_{s} / N_{0}$ values that we investigated in our experiment. This explains why the minimal distortions of our two design algorithms are roughly the same at all $E_{s} / N_{0}$ values. Specifically, the effect of channel errors is negligible on the total distortion at the optimal bit allocation, and for the optimal bit allocation the channel bit-error-probabilities of the optimal RCPC channel code are approximately zero. Therefore, since the source code of VQ-RCPC is designed for a perfect channel, this code design is approximately optimal and has
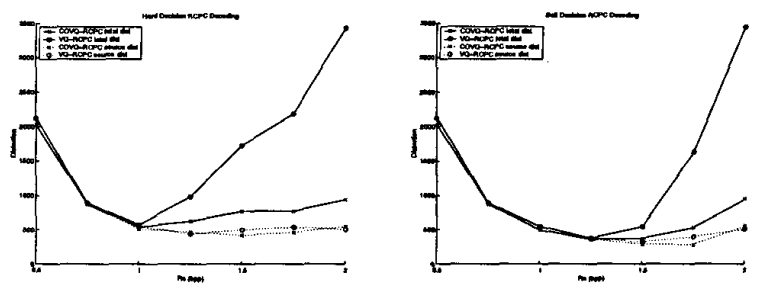

Figure 7: Distortion versus $R_{s}$ at $E_{s} / N_{0}=10 \mathrm{~dB}$.

similar performance as the optimal iteratively designed COVQ-RCPC.

\section{Summary}

We describe two joint source and channel code techniques for Rayleigh fading channels. The first technique (VQ-RCPC) is a sequential design consisting of a VQ designed for a perfect channel followed by a RCPC channel code, where the channel code is designed relative to the VQ to minimize end-to-end distortion. The second technique (COVQ-RCPC) combines COVQ with an RCPC channel code in an iterative design procedure. Both code designs optimize the bit allocation between the source and channel coders. The optimal bit allocation reduces distortion by up to $9 \mathrm{~dB}$ relative to a sub-optimal bit allocation. Our joint code designs also reduce distortion by up to $6 \mathrm{~dB}$ relative to standard COVQ. The distortion of both proposed code designs are approximately the same. That is because the optimal bit allocation for each code design results in a channel code that removes most of the channel errors, in which case both of the design procedures are roughly equivalent. Thus, the most important aspect for any of these source and channel code designs is the optimal bit allocation between the source and channel coders.

\section{References}

[1] A. Goldsmith and M. Effros, "Joint design of fixed-rate source codes and multiresolution channel codes." IEEE Trans. Commun., Vol. 46, No. 10, pp. 1301-1312, Oct. 1998.

[2] Hai-Xin Tie, A. Goldsmith and M. Effros, "Joint design of fixed-rate source codes and UEP channel codes for fading channels," Proc. Asilomar Conf. on Signals, Systems and Computers, Oct. 1998.

[3] N. Farvardin and V. Vaishampayan, "On the performance and complexity of channel-optimized vector quantizers," IEEE Trans. Inform. Theory, Vol. 37, pp. 155-160, Jan. 1991.

[4] J. Hagenauer, "Rate-compatible punctured convolutional codes (RCPC codes) and their applications," IEEE Trans. Commun., Vol. 36, No. 4, pp. 389-400, April 1988. 